\title{
Phase hologram formation in highly concentrated phenanthrenequinone-PMMA media
}

\author{
U V Mahilny ${ }^{1}$, D N Marmysh ${ }^{1}$, A L Tolstik ${ }^{1}$, V Matusevich ${ }^{2}$ and \\ R Kowarschik ${ }^{2}$
}

${ }^{1}$ Physical Department of Belarusian State University, Nezavisimosti Avenue 4, 220030 Minsk, Belarus

${ }^{2}$ Institute of Applied Optics, Friedrich-Schiller-University Jena, Max-Wien-Platz 1, 07743 Jena, Germany

E-mail: mogilny@bsu.by

Received 12 March 2008, accepted for publication 30 May 2008

Published 20 June 2008

Online at stacks.iop.org/JOptA/10/085302

\begin{abstract}
For phase holographic gratings in layers of polymethylmethacrylate, containing phenanthrenequinone in high concentration (nearly $3 \mathrm{~mol} \%$ ), a discrepancy between experimental (up to 9) and estimated ( 45) magnitudes of the thermal diffusion amplification coefficient has been revealed. Analysis of plausible reasons of the lower experimental efficiency of the diffusion amplification has been carried out. The influence of material deformations on the reflection grating formation process was investigated experimentally. It is shown that thermoactivated amplification of holograms under high phenanthrenequinone concentration and its profound modulation are depressed by the arising density 'grating'.
\end{abstract}

Keywords: holography, holographic recording media, phase holographic grating, phenanthrenequinone, polymethylmethacrylate

\section{Introduction}

Polymeric materials for recording of phase holograms with thermal amplification using phenanthrenequinone (PQ) as a photosensitive component have been known since the beginning of the 1990s [1]. The postexposure hologram amplification in such recording media is caused by the diffusion of residual photosensitive molecules leading to a homogeneous distribution of their concentration. Some factors precluding maximum values of the diffusion amplification factor to be attained have been established: incomplete photolinkage of $\mathrm{PQ}$ to the polymeric matrix, and semiquinone radical diffusion [1-3]. A postexposure behavior of holographic gratings has been studied in detail over a wide temperature interval and at different thermal treatment times [3]. The experimentally obtained patterns of hologram transformations are in accord with the knowledge of the principal role played by PQ diffusion in the amplification process $[1,3]$. Degradation of holograms is associated with a diffusive motion of macromolecules and macromolecular segments including the added photoproducts [4-6]. The general expression for the modulation amplitude kinetics of the refractive index in the process of thermally activated hologram transformations in the PQ-containing medium has been proposed [5]. The characteristics of the PQ-based material and other phase holographic materials have been compared [7].

However, the majority of studies into the recording materials with PQ involve media with low PQ contents $(\sim 0.5 \mathrm{~mol} \%)$ using 'weak' holograms with an insignificant modulation depth of the refractive index. And the formation of high-efficiency holograms in relatively thin PQcontaining recording layers is commonly associated with PQ concentrations of about a few per cent [8]. Deeper modulation of the PQ concentration in this case may contribute to a more complex process of hologram formation, e.g. due to the photoinduced material deformations [9-11].

The principal objective of this work is to determine the factors limiting the diffraction efficiency of holograms within the layers of polymethylmethacrylate-phenanthrenequinone 
(PMMA-PQ) at high concentrations of PQ. Because of the deformations that are liable to occur in the process of a hologram formation in these conditions, considerable attention has been devoted to volume reflection holograms, where the deformation phenomena seemed to meet with the least difficulties.

\section{Estimation of hologram parameters}

Phase recording in a polymeric material with PQ is based on its ability to attach to the polymeric chains under the effect of light. Recording of a phase holographic grating in this material may be schematically demonstrated as follows. In the process of recording, two distributions are produced for the concentrations of the attached photoproduct and unreacted PQ. The first is in phase with the spatial light distribution within the layer, the latter being antiphase. Each of the concentration distributions is associated with the respective contribution into the distribution of the refractive index. The modulation amplitude of the latter may be determined by the Lorentz-Lorenz [12] formula as follows:

$$
\Delta n=\frac{\left(n^{2}+2\right)^{2}}{6 n}\left(R_{\mathrm{p}} \Delta C_{\mathrm{p}}-R_{\mathrm{pq}} \Delta C_{\mathrm{pq}}\right),
$$

where $n$ is the average refractive index of the layer, $R_{\mathrm{p}}$ and $R_{\mathrm{pq}}$ the molar refractions of the photoproduct and $\mathrm{PQ}$, respectively, and $\Delta C_{\mathrm{p}}$ and $\Delta C_{\mathrm{pq}}$ the modulation amplitudes for the photoproduct and PQ concentrations.

The postexposure thermal treatment results in a diffusion flattening of the PQ distribution in the layer, a contribution of the unreacted PQ to the refractive index modulation degrading. As this takes place, the photoproduct distribution remains practically invariable due to its links with macromolecules. Then the regularity in variation of the total modulation amplitude of the refractive index may be given by the expression that follows from the solution for a diffusion equation with the initial sine distribution of the concentration:

$$
\Delta n=\frac{\left(n^{2}+2\right)^{2}}{6 n}\left[R_{\mathrm{p}} \Delta C_{\mathrm{p}}-R_{\mathrm{pq}} \Delta C_{\mathrm{pq}}\left(t_{\mathrm{e}}\right) \mathrm{e}^{-\gamma\left(t-t_{\mathrm{e}}\right)}\right],
$$

where $\Delta C_{\mathrm{pq}}\left(t_{\mathrm{e}}\right)$ is the light field induced modulation amplitude for the concentration of photosensitive molecules, $\gamma=$ $D(2 \pi / d)^{2}$ the rate constant of the amplification process, $D$ the diffusion coefficient, $d$ the holographic grating period, $t$ the thermal treatment time of the layer, and $t_{\mathrm{e}}$ the recording time of a holographic grating. The final distribution of the refractive index is determined by the photoproduct distribution:

$$
\Delta n=\frac{\left(n^{2}+2\right)^{2}}{6 n} \Delta C_{\mathrm{p}} R_{\mathrm{p}}
$$

The diffusion amplification coefficient $M$, defined as the ratio of $\Delta n(\infty)$ when amplification is terminated to $\Delta n\left(t_{\mathrm{e}}\right)$, attained upon recording, is found from the following expression:

$$
M=\frac{\Delta n(\infty)}{\Delta n\left(t_{\mathrm{e}}\right)}=\frac{R_{\mathrm{p}}}{\Delta R},
$$

where $\Delta R=R_{\mathrm{p}}-R_{\mathrm{pq}}$ is the difference between values of the refractions of the photoproduct and PQ. The expression (4) is correct assuming that all the photoproduct molecules are linked to the macromolecules:

$$
\Delta C_{\mathrm{p}}=\Delta C_{\mathrm{pq}}\left(t_{\mathrm{e}}\right)
$$

As follows from equations (3) and (4), the final value of $\Delta n$ is determined by the photoproduct concentration modulation and by its molar refraction, whereas the amplification factor $M$ is only dependent on the photoproduct refraction and the difference between values of refractions of the photoproduct and PQ. The photoproduct refraction estimated by the bond refractions [13] is $R_{\mathrm{p}}=62.9 \mathrm{~cm}^{3} \mathrm{~mol}^{-1}$. The expression (1) and experimental data of the modulation concentration of the photoproduct and the reachable value $\Delta n$, taken from [14], have been used to estimate $\Delta R \approx 1.4 \mathrm{~cm}^{3} \mathrm{~mol}^{-1}$. Considering this value and expression (4) the amplification factor calculations result in $M \approx 45$. For the layer containing $3 \mathrm{~mol} \%$ of $\mathrm{PQ}$, on condition that $\Delta C_{\mathrm{p}}$ comes to $100 \%$ of the PQ concentration within the layer, the value of $\Delta n$ after the diffusion amplification process termination should be equal to 0.04 . However, from the experimental data it follows that an amplification factor for volume transmission gratings is never over 9, and the final modulation amplitude of the refractive index is below $2 \times 10^{-3}$.

During analysis of the recording process used to form a holographic grating, of particular importance is the real modulation amplitude of the photoproduct concentration attainable in the process of recording. It may be limited by the form of kinetics determining the PQ consumption in the process of recording. The effect of PQ consumption pattern on the form of the profile characteristics for the holographic grating refractive index in the process of its recording has been analyzed [14]. It has been found that nonlinearity of the PQ consumption curve is responsible for deviation of the profile from the sinusoidal form determined by the light field. This leads to the appearance of higher-order diffraction as well as to limited modulation amplitudes of the refractive index $\Delta n$ for the main diffraction order. On exposures characteristic for hologram recording in polymeric layers with a high content of PQ, $\Delta n$ of the main grating comes to only $50 \%$ of the maximum value possible at $100 \%$ phototransformation of PQ. Because of this, the difference in the calculated and experimental values of $\Delta n$ is somewhat reduced, though still being significant. In this way the experimental results and theoretical calculations differ significantly. It is obvious that such a difference is due to some phenomena that are omitted in the simplified calculations. We have considered them to be the main reason for the discrepancy discussed. The results of the investigations are given below.

\section{Experimental details}

Recording layers $80-110 \mu \mathrm{m}$ in thickness were prepared by pouring the liquid solution of PMMA and PQ (2.5-3 mol\%) onto a glass plate with subsequent drying [8]. Phase volume reflection gratings (RGs) with the period $d=0.24 \mu \mathrm{m}$ and insignificant groove tilt $\beta=0.7^{\circ}-2.7^{\circ}$ were recorded 

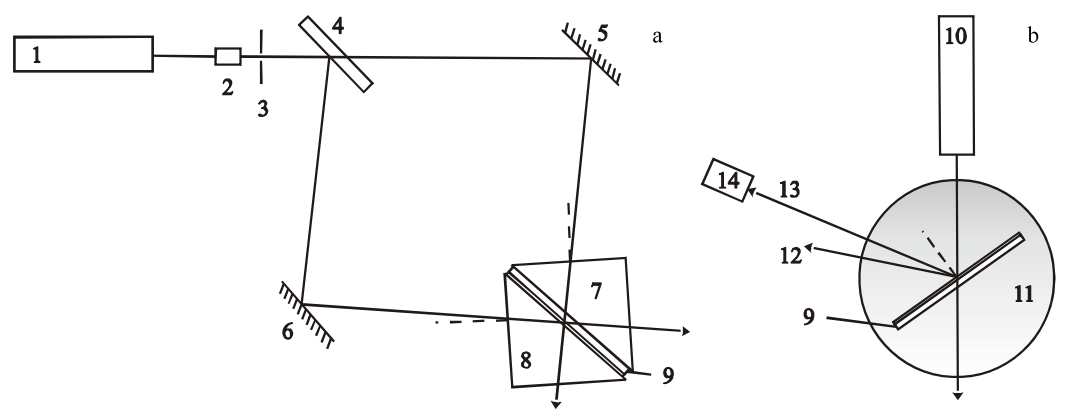

Figure 1. Scheme of the experimental set-up for recording (a) and reconstruction (b) of the reflection gratings. 1-argon laser, 2 -collimator, 3-diaphragm, 4-beam splitter, 5 and 6-mirrors, 7 and 8-right-angle prisms, 9-glass substrate with the recording layer, 10- $\mathrm{He}-\mathrm{Ne}$ laser, 11-precision rotation stage, 12-reflected beam, 13-diffracted beam, 14-photodetector.

in counterpropagating beams using the asymmetric scheme (figure 1(a)). Recording was realized with the use of argon laser radiation at the wavelengths 488 and $514.5 \mathrm{~nm}$. The recording exposures of the gratings were $3-10 \mathrm{~J} \mathrm{~cm}^{-2}$. The required parameters of the $\mathrm{RG}$ were provided under recording due to the use of right-angle prisms, having the refractive index of 1.5 , as the radiation coupling elements.

Subsequent to $\mathrm{RG}$ recording, the samples were thermally treated at 55,80 , and $110^{\circ} \mathrm{C}$. The first temperature is considerably lower than that of the polymer vitrification (about $100{ }^{\circ} \mathrm{C}$ for pure PMMA), the second temperature being close to and the third one being in excess of this characteristic temperature. Between the annealings, the samples were kept at room temperature for $10 \mathrm{~min}$, following which measurements of the angular selectivity contours of the RG (the diffraction efficiency $\eta$ as a function of the incidence angle of a probe beam on the layer surface) were done using the set-up presented in figure 1(b). A He-Ne laser $(\lambda=633 \mathrm{~nm})$ was used as a probe. The diffraction efficiency was determined as the intensity ratio of the diffracted and incident beams. Using the maximal $\eta$ values from the angular selectivity contour the modulation amplitude for the refractive index $\Delta n$ was found by the formula for unslanted phase volume reflection gratings [15]:

$$
\Delta n=\operatorname{arcth}\left(\frac{\lambda \cos (\pi / 2-\theta)}{\pi h} \sqrt{\eta}\right),
$$

where $\theta$ is the Bragg angle and $h$ is the layer thickness.

The surface elevation height of the exposed regions was determined with the help of an interference microscope. Homogeneous exposure of the RG was performed for the samples using Ar laser radiation with the wavelength $514.5 \mathrm{~nm}$ at different stages of thermal treatment at temperatures 55 and $80^{\circ} \mathrm{C}$. According to the data of [14], the exposure energy was in excess of $300 \mathrm{~J} \mathrm{~cm}^{-2}$, offering practically complete phototransformation of PQ in the polymeric layer.

\section{Results and discussion}

Figure 2 presents the typical curves for the refractive index modulation amplitude as a function of annealing time at temperatures of $55^{\circ} \mathrm{C}$ (curve 1, curve 2 for $t \leqslant 6.6 \mathrm{~h}$ ) and $80^{\circ} \mathrm{C}$

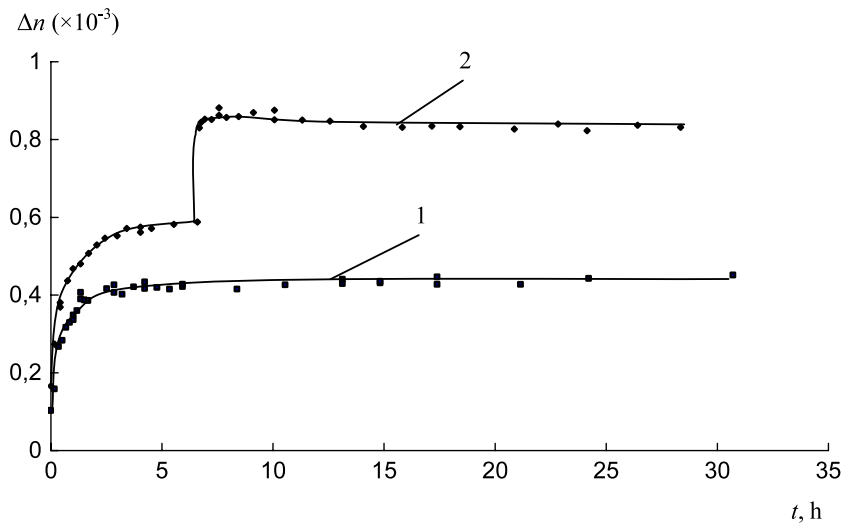

Figure 2. The refractive index modulation amplitude as a function of the thermal treatment time at $55^{\circ} \mathrm{C}$ (curve 1 , curve 2 for $t \leqslant 6.6 \mathrm{~h}$ ) and $80^{\circ} \mathrm{C}$ (curve 2 for $t>6.6 \mathrm{~h}$ ).

(curve 2 for $t>6.6 \mathrm{~h}$ ). Heating at $55^{\circ} \mathrm{C}$ reveals an increase in $\Delta n$. Curve 1 exhibits two sections with different rates of rise. For the first section the rise rate of $\Delta n$ is much greater than for the second one. An additional rise in $\Delta n$ occurs after heating at $80^{\circ} \mathrm{C}$ (curve 2 ).

In the process of thermal treatment at a temperature of $55^{\circ} \mathrm{C}$ no significant changes in the form and position of the angular selectivity profile have been observed. Besides, no surface deformations have been revealed within the bounds of holographic gratings. When the temperature was elevated to $80^{\circ} \mathrm{C}$, simultaneously with an additional growth of $\Delta n$, a shifting of the angular selectivity contour to greater diffraction angles was initially observed. It is followed by a subsequent monotonic decrease of the diffraction angles. In the process of thermal treatment the shape of the angular selectivity contour was practically invariable. Figure 3 shows $\Delta n$ (curve 1) and changes in $\alpha_{\max }\left(\Delta \alpha_{\max }\right)$, associated with a maximum of the diffraction efficiency (curve 2) as a function of the annealing time at $80^{\circ} \mathrm{C}$. The sample was preliminarily treated at a temperature of $55^{\circ} \mathrm{C}$ for a period of $6.6 \mathrm{~h}$. After the initial increase of $\Delta n$ and $\alpha_{\max }$ at the first heating stage, these parameters decreased monotonically. Also, several annealing steps at $80^{\circ} \mathrm{C}$ have caused the formation of surface swellings on the layer in the limits of holographic gratings. The initial swelling height of $0.2 \mu \mathrm{m}$ has increased to nearly $0.3 \mu \mathrm{m}$ (figure 4). 


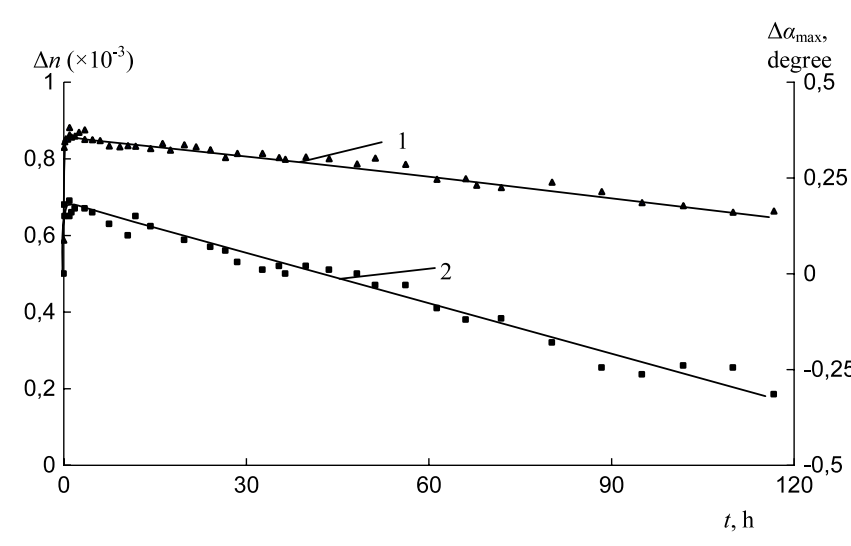

Figure 3. Kinetics of $\Delta n$ (1) and change of the diffraction angle $\alpha_{\max }(2)$ as a function of the annealing time at $80^{\circ} \mathrm{C}$. Preliminary thermal treatment of the layer over a period of $6.6 \mathrm{~h}$ at $55^{\circ} \mathrm{C}$.

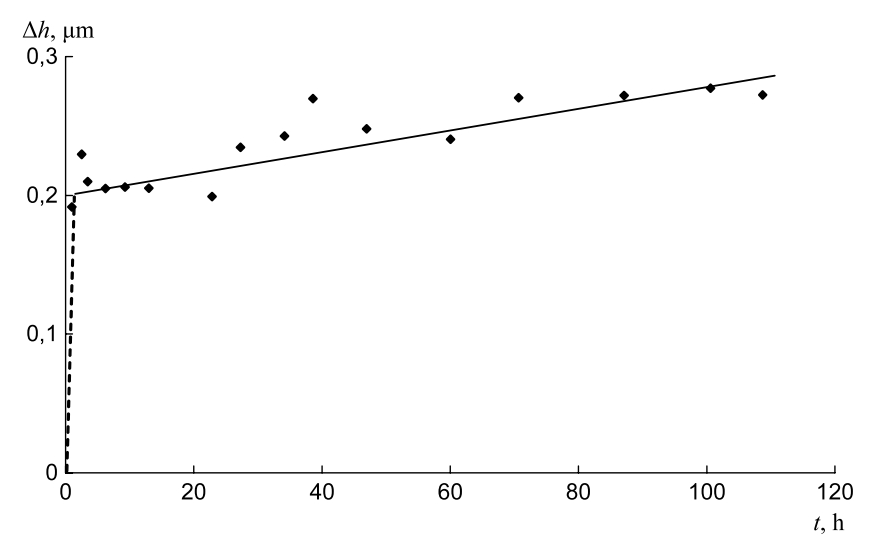

Figure 4. The swelling height as a function of the annealing time at $80^{\circ} \mathrm{C}$.

Figure 5 shows the typical curves for $\Delta n$ and $\Delta \alpha_{\max }$ as a function of the annealing time at a temperature of $110^{\circ} \mathrm{C}$. The layer was preliminarily subjected to thermal treatment at $80^{\circ} \mathrm{C}$ for $32 \mathrm{~h}$. Unlike the situation on going from 55 to $80^{\circ} \mathrm{C}$, a rise in the temperature has caused no initial increase of $\Delta n$ and $\alpha_{\max }$. The values of $\Delta n$ and $\alpha_{\max }$ monotonically decreased with the thermal treatment time. At the same time, the swelling height was increasing (figure 6).

The rapidly growing sections of $\Delta n$ kinetics at $55^{\circ} \mathrm{C}$ are reasonably approximated by a function of the form $\Delta n=$ $a_{1}-a_{2} \exp (-\gamma / t)$, following from expression (4). This points to a diffusion nature of increase in the modulation amplitude. Because of the presence of slow $\Delta n$ kinetics at $55^{\circ} \mathrm{C}$ and of a considerable increase in $\Delta n$ with temperature elevation to $80^{\circ} \mathrm{C}$, it is of interest what is the degree of PQ diffusion completeness. To estimate the PQ modulation depth within the layer at different sections of the amplification curve of $\Delta n$, it is convenient to expose the grating to a spatially homogeneous light field. Provided the diffusion process is completed, i.e. the PQ distribution within the layer is homogeneous, the light field exposure should result in changes only of the average refractive index of this layer. As this takes place, the value of $\Delta n$ should remain constant. Homogeneous exposure of a holographic grating immediately after recording should lead to its complete

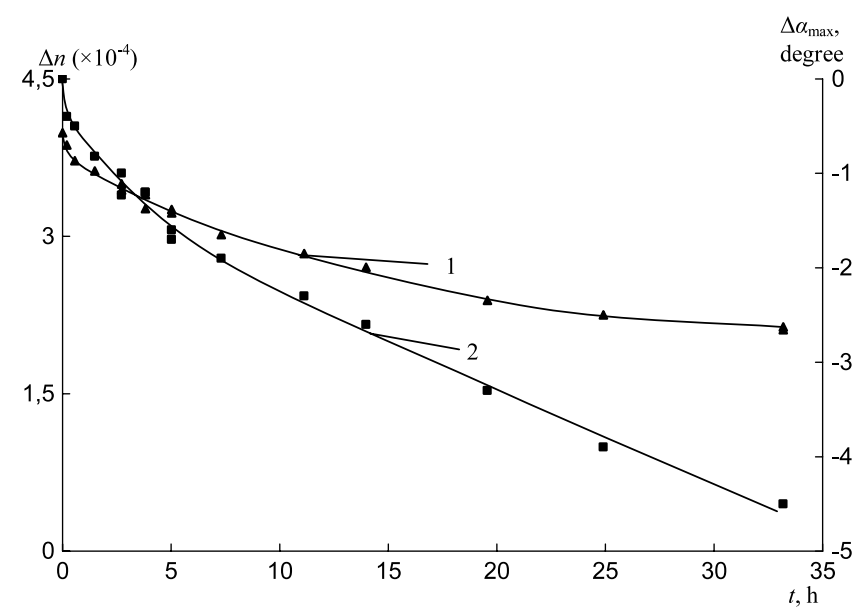

Figure 5. Kinetics of $\Delta n(1)$ and the change of the diffraction angle $\alpha_{\max }$ (2) as a function of the annealing time at $110^{\circ} \mathrm{C}$. Preheating of the layer over a period of $32 \mathrm{~h}$ at $80^{\circ} \mathrm{C}$.

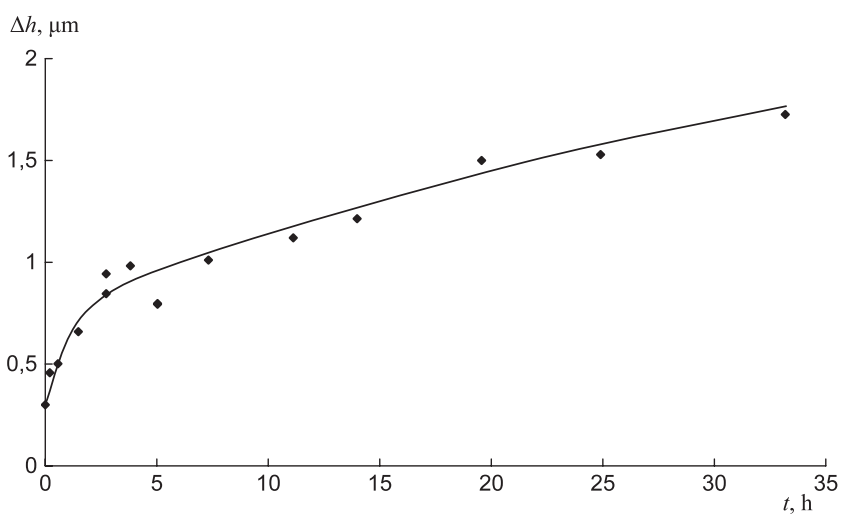

Figure 6. The swelling height as a function of the annealing time at $110^{\circ} \mathrm{C}$.

erasing. To describe the completeness degree of PQ diffusion, it is convenient to introduce the coefficient $K$, as follows:

$$
K=\frac{\Delta n(H=0)-\Delta n(H)}{\Delta n\left(t_{\mathrm{e}}\right)},
$$

where $H$ is exposure under homogeneous irradiation.

It is easily seen that $K$ represents a relative modulation amplitude for the PQ concentration at the point of the annealing curve, where a homogeneous exposure of the grating is performed. Possible values of $K$ are found over the range $0 \leqslant K \leqslant 1$. Diffusion of PQ may be considered complete when $K$ is close to 0 .

Figure 7 demonstrates $\Delta n$ (curve 1) and coefficient $K$ (curves 2, 3) as a function of the annealing time at $55^{\circ} \mathrm{C}$ (curve 2), and also after the temperature is elevated to $80^{\circ} \mathrm{C}$ with a $40 \mathrm{~min}$ heating prior to the homogeneous exposure procedure (curve 3 ). The coefficient $K$ is decreased from 1 to $\sim 0.3$ during the time period associated with the section of rapidly increasing $\Delta n$, remaining practically constant afterward. For the gratings subjected to thermal treatment at $80^{\circ} \mathrm{C}, \mathrm{K}$ is very close to zero. 


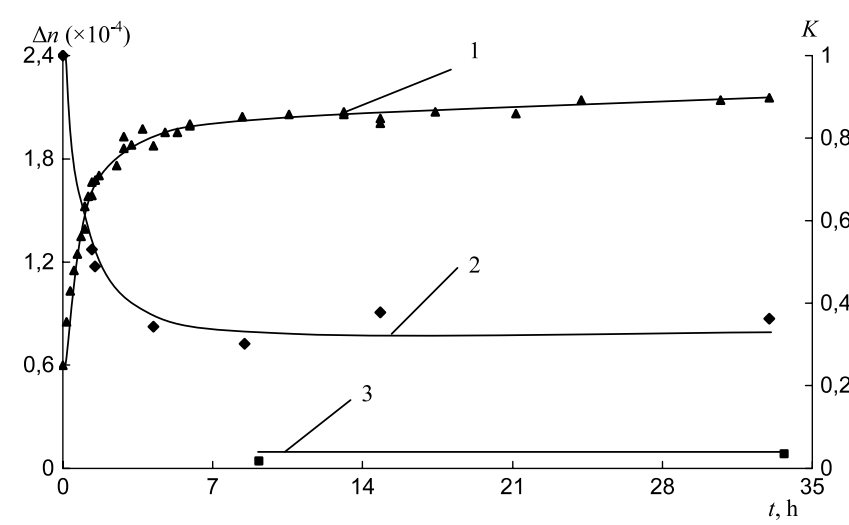

Figure 7. Dependences of $\Delta n(1)$ and $K$ factor $(2,3)$ on the annealing time at $55^{\circ} \mathrm{C}$ before the homogeneous exposure. In the case of curve 3 , subsequent to annealing at $55^{\circ} \mathrm{C}$, an additional thermal treatment was conducted at $80^{\circ} \mathrm{C}$ for $40 \mathrm{~min}$.

Based on the obtained results, one can infer that at a temperature of $55^{\circ} \mathrm{C}$ the section of rapidly growing $\Delta n$ is in fact of a diffusion character. However, at this temperature such a 'high-rate' diffusion of PQ does not result in homogeneity of its distribution. Further increase in $\Delta n$ occurs due to a 'slow' process. Unfortunately, our experimental data fail to make positive conclusions concerning its nature and effect on the PQ distribution (figure 7, curves 1 and 2). It is necessary to note that the account of the unfinished PQ diffusion cannot neutralize the difference in experimental data and theoretical calculations. With the annealing temperature increased to $80^{\circ} \mathrm{C}, \Delta n$ is growing (figure 2, curve 2), making the PQ distribution practically homogeneous in a short time (figure 7 , curve 3). No doubt this takes place because of the PQ diffusion. The characteristic values of amplification factor for an RG subjected to thermal treatment at $80^{\circ} \mathrm{C}$ are about 56. In similar conditions (PQ concentration, exposure during recording process) transmission holographic gratings exhibit similar values of $M$ [16]. So, for an RG the difference in experimental data and theoretical calculations is approximately the same as in case of transmission gratings.

By equality (5) laying the basis for the calculations of $M$ it is assumed that $\mathrm{PQ}$ is completely transformed into the photoproduct linked to the polymeric matrix. But it is well known that in polymeric layers produced by block polymerization only about $50 \%$ of the total quantity of the photoreduced PQ molecules are attached to the macromolecules [17]. It is supposed that some portion of the molecules is interacting with a residual monomer, forming low-molecular photoproducts. Obviously, these photoproducts make no contribution to the final distribution of the refractive index, which may lead to a considerable decrease in $\Delta n$ and $M$. At the same time, in the layers under study, which were prepared by pouring, we have used PMMA purified of the monomer. And the method used for the preparation of recording layers is responsible for considerable amounts of the residual solvent in these layers whose interactions with PQ could not be excluded. Besides, due to a high concentration, the possibility of the photoreaction between PQ molecules is not ruled out. In both cases low-molecular photoproducts

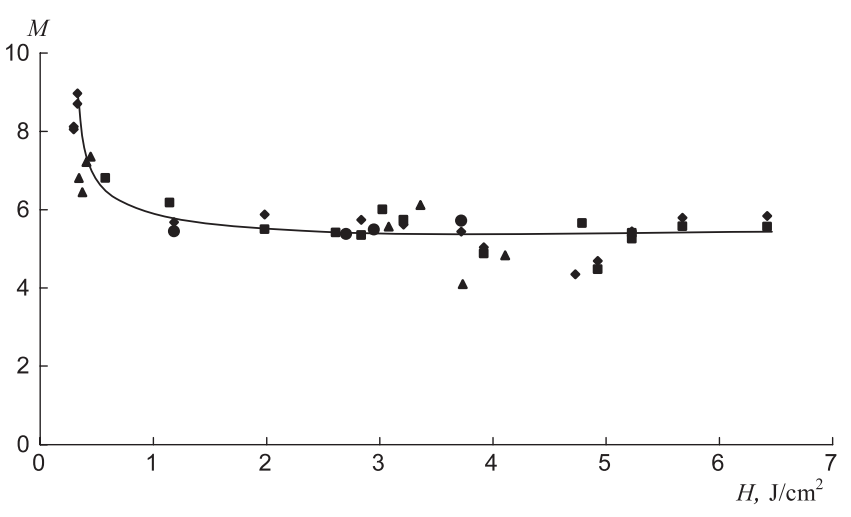

Figure 8. The amplification factor of transmission gratings as a function of exposure. Grating period $d=0.5$ (circles), 1 (diamonds), 2 (squares), and $3 \mu \mathrm{m}$ (triangles). The temperature of treatment is $80^{\circ} \mathrm{C}$.

may be formed. As demonstrated by the experiments, a degree of PQ linking to PMMA within the layers similar to those described is, however, close to $100 \%$ [18]. This makes it possible to exclude the formation of low-molecular photoproducts as a cause of the discrepancy between the calculated and experimental values of $\Delta n$ and $M$. Also, these values may be decreased due to nonlocality of the photolinkage reaction between PQ and the polymer. The first stage of this reaction is the photoreduction of PQ with the formation of a semiquinone radical, and the second stage is recombination of the latter with the macroradical (strictly speaking, linkage) [17]. A shift of the semiquinone radical until the moment of its linkage at a rather long lifetime may result in a decrease of $\Delta C_{\mathrm{p}}$ and hence $\Delta n$ and $M$.

It is clear that in the case when the radical diffusion has a significant effect on hologram amplification, the relationship between the amplification factor $M$ and period of a holographic grating should be exhibited. Figure 8 shows the amplification factor as a function of exposure for transmission gratings with different periods. The curve is constructed on the basis of our previous results [16] and more recent unpublished data. Figure 8 demonstrates the absence of the expected dependence of the amplification factor on the period, at least for transmission gratings. As is seen, the amplification factor increases as the exposure is reduced in the process of recording. This pattern is hardly associated with any of the abovementioned factors limiting $M$ and $\Delta n$.

The experimental data presented point to the developing inhomogeneous deformations of the RG under thermal treatment at $80^{\circ} \mathrm{C}$ and higher temperatures (figures 3-6). During the period of annealing $\left(80^{\circ} \mathrm{C}\right)$ that is on the order of the amplification time ( $\sim 2 \mathrm{~min})$, the swelling height increases to $\sim 0.2 \%$ of the layer thickness (figure 4 ). In parallel, an increase of $\alpha_{\max }$ occurs (figure 3, curve 2).

The angle $\alpha_{\max }$ is related to the parameters of a holographic grating and recording layer by the following expression:

$$
\alpha_{\max }=\arcsin (n \cos (\theta+\beta)),
$$


where $\beta$ is the groove tilt, and $\theta$ the angle determined by the Bragg condition:

$$
\sin \theta=\frac{\lambda}{2 n d},
$$

$\lambda$ being the wavelength of the probe radiation.

According to expression (8), the change in the angle $\alpha_{\max }$ may be due to variations in the period of a holographic grating $d$, groove tilt $\beta$, and average refractive index $n$ on extension or shrinkage of the photoconverted material. Considering the deformation of the RG as developing normally to the layer plane and the extension factor of the material to be proportional to the photoproduct concentration, one can easily show the validity of the following expressions:

$$
\begin{gathered}
\frac{\delta \rho}{\rho}=-\frac{\Delta h}{h}=-\frac{\Delta d}{d}, \\
\delta n=-\frac{\left(n^{2}+2\right)\left(n^{2}-1\right)}{6 n} \frac{\Delta h}{h}, \\
\delta \beta=\operatorname{arctg}\left(\frac{h+\Delta h}{h} \operatorname{tg} \beta\right)-\beta,
\end{gathered}
$$

where $\rho, h, d$ are the mean values of the material density, the layer thickness and the grating period; $\delta \rho$ the variation of average density, $\Delta h, \Delta d$ variations of thickness and period, and $\delta \beta$ the variation of groove tilt. Expression (11) describes the contribution made by the density changes to variations of the average refractive index.

Figure 9 shows the calculated curve for $\Delta \alpha_{\max }$ as a function of the changing thickness of a recording layer, constructed with the use of expressions (8)-(12). As is seen, the angle $\alpha_{\max }$ should be increased with a growing thickness of the grating. Thus one can conclude that the initial swelling is really caused by dilatation of the photoconverted material (figures 3 and 4). In the case of a prolonged thermal annealing $\alpha_{\max }$ is decreased, whereas the swelling is still growing. Similar behavior has been exhibited by the RG at $110^{\circ} \mathrm{C}$ (figures 5 and 6 ). These features may be attributed to general shrinkage of the polymeric matrix under the prolonged thermal effect. And it seems to be natural that the lower the photoconversion extent of the material the higher its shrinkage rate. This dependence leads to a decrease of $\Delta n$ under longtime heating (figure 5, curve 1).

So the inhomogeneous dilatation of the grating structure is able to bring about modulation of the material density (density grating), whose contribution to the refractive index modulation is opposite to that of the photoproduct distribution. As a consequence of these deformations the refraction index modulation amplitude $\Delta n$ and amplification factor $M$ should be decreased in comparison with the values obtained by the estimations. Nevertheless this attractive and qualitatively consistent hypothesis seems insufficient. Indeed we did not observe any swelling formation at $55^{\circ} \mathrm{C}$, but combining expression (2), expression (11) adapted for the modulation amplitude of $n$, and taking into account the extent of the diffusion completeness (figure 2), one can easily show that $\Delta h$ has to be on the order of several tenths of a micron to ensure discrepancies existing between the experimental and estimated magnitudes of $\Delta n$ and $M$. Therefore we

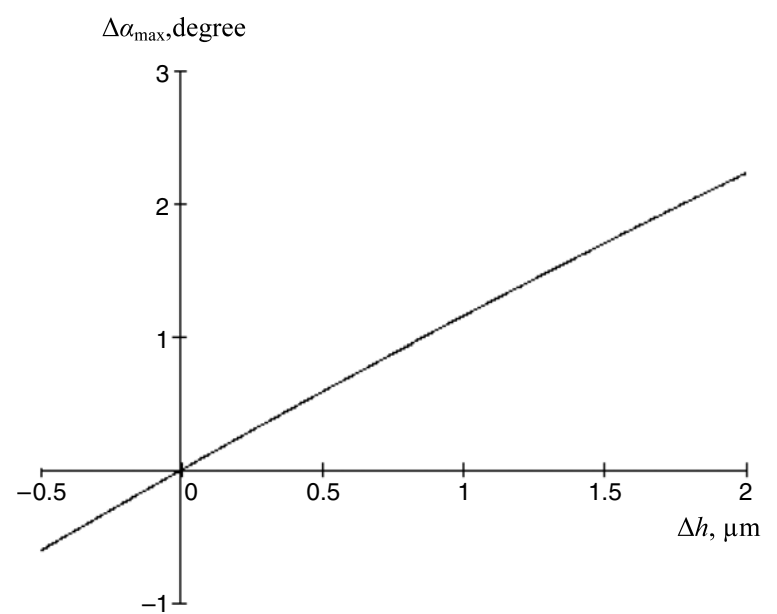

Figure 9. Changes in the angle $\alpha_{\max }$ as a function of the recording layer thickness change $\Delta h$.

are inclined to suppose that 'antiphase' density grating is formed by at least two processes: dilatation of photoconverted material mentioned above and some additional relaxation process of diffusive nature synchronized to the PQ diffusion and so indistinguishable against its background. This last process might be a diffusion of free volume in the line of PQ diffusion [19] or oppositely directed diffusion of residual solvent [20] or both.

The experimental results presented reveal some features for the thermal amplification of holographic gratings within the layers of PMMA with a high content of PQ and deep modulation of its concentration. The thermal treatment at a temperature that is close to the material vitrification temperature provides a homogeneous concentration of the residual PQ. However, the process of PQ diffusion is accompanied by the 'antiphase' density grating formation, weakening the effect of diffusive amplification and not allowing attainment of the calculated values of the refractive index modulation depth and amplification factor. The density grating is formed by inhomogeneous expansion of the photoconverted material and is seemingly a process of diffusive nature (free volume diffusion, diffusion of residual solvent).

Elevation of the annealing temperature results in general shrinkage of the material, additionally decreasing the real values of $\Delta n$ and $M$. Provided thermal amplification is realized at a temperature that is considerably lower than the material vitrification temperature, it is impossible to attain a homogeneous concentration of the residual PQ during a reasonable time.

\section{Conclusion}

Thus, the work presents an analysis of the factors limiting the diffraction efficiency of holograms within the layers of polymethylmethacrylate-phenanthrenequinone at a high content of phenanthrenequinone. It is demonstrated that using the theoretical estimates for holographic gratings in the photorecording material containing up to $3 \mathrm{~mol} \%$ of PQ the values of the diffusive amplification factor and refractive index modulation amplitude are $\sim 45$ and 0.02 , respectively. 
The experimental values of these parameters both for volume transmission and reflection gratings are considerably lower. Based on the results obtained, we can exclude the low efficiency of photolinkage and diffusion of semiquinone radicals as the principal cause of this discrepancy.

In the case of the reflection holographic gratings under study, at an optimum temperature $\left(\approx 80^{\circ} \mathrm{C}\right)$ close to the vitrification temperature, the diffusion process is fully completed over the period of thermal amplification. However, in these conditions the differences between the calculated and experimental amplification factors and refractive index modulation amplitudes are also revealed. It is believed that this is caused by formation of the material density 'grating' compensating for the effects of the 'grating' due to the photoproducts.

\section{References}

[1] Veniaminov A V, Goncharov V F and Popov A P 1991 Opt. Spectrosc. 70505

[2] Veniaminov A V, Bartsch E and Popov P 2005 Opt. Spectrosc. $99776-82$

[3] Veniaminov A and Sedunov Y 1996 Polym. Sci. 3859

[4] Veniaminov A V, Sedunov Y N, Popov A P and Bandyuk O V 1996 Opt. Spectrosc. 81617

[5] Veniaminov A V and Bartsch E 2006 Opt. Spectrosc. $101290-8$

[6] Veniaminov A and Sillescu H 1999 Macromolecules 32 1828-37
[7] Steckman G J, Solomatine I, Zhou G and Psaltis D 1998 Opt. Lett. 23 1310-2

[8] Mahilny U V, Marmysh D N, Stankevich A I, Tolstik A L, Matusevich V and Kowarschik R 2006 Appl. Phys. B 82 299-302

[9] Mogil'nyi V V, Gritsai Y V and Kovalev S V 1999 Tech. Phys. 44 941-4

[10] Dhar L, Melinda G S, Wysocki T L, Bair H, Schilling M and Boyd C 1998 Appl. Phys. Lett. 73 1337-9

[11] Pen E F, Rodionov M Y and Shelkovnikov V V 2006 J. Opt. Technol. 73 475-9

[12] Born M and Wolf E 1968 Principles of Optics (Oxford: Pergamon)

[13] Ioffe B V 1995 Chimicheskaja Enciklopedija vol 4, ed N S Zefirov et al (Moskva: Bolshaja rossijskaja enciklopedija) p 262 (in Russian)

[14] Marmysh D N, Mahilny U V and Tolstik L A 2006 Izv. Ross. Akad. Nauk. Ser. Fiz. (Bull. Russ. Aca. Sci.: Phys.) 70 1796-9 (in Russian)

[15] Kogelnik H 1969 Bell Syst. Tech. J. 48 2909-47

[16] Mahilny U V, Marmysh D N, Tolstik A L, Matusevich V and Kovarschik R 2005 Proc. SPIE 5956 342-8

[17] Banduk O, Shelehov N, Popov A and Danilova M 1988 J. Prikladnoi Khimii (Russian J. Appl. Chem.) 61 946-8 (in Russian)

[18] Marmysh D N, Stankevich A I and Mahilny U V 2007 Proc. 7th Int. Conf. IRS-2007 (Minsk) pp 47-9

[19] Rostiashwili V G, Irshak V I and Rozenberg B A 1987 Steklovanie Polimerov (Leningrad: Chimiya) (in Russian)

[20] Marmysh D N and Mahilny U V 2005 Vestn. BGU Seriya 11 21-6 (in Russian) 